\title{
The Substantiation of the Optimal Parameters for Dough Pinning-Out Rollers
}

\author{
Tetiana Vitenko ${ }^{* *}$, Igor Stadnik', Paweł Droździel ${ }^{2}$, Anna Rudawska² \\ 1 Ivan Puluj TNTU, Faculty of Engineering of Machines, Structures and Technologies, Ruska 56 Street, \\ 46001 Teronpil, Ukraine \\ 2 Lublin University of Technology, Faculty of Mechanical Engineering, ul. Nadbystrzycka 36, 20-618 Lublin, \\ Poland \\ * Corresponding author's e-mail: tetiana.vitenko@gmail.com
}

\begin{abstract}
The article defines the rather complex and unpredictable movement of the dough in the gap between the working rolls and suggests the calculation for the optimal parameters for the rolls shape (particularly the parameters and their effect on the dough). The functional dependences of the permissible diameter and the gap between rotating rollers were substantiated. The block diagram of the geometric bounding parameters of the gradient field of the viscous fluid flow was proposed and the equation on the basic constructive parameters ( $h, r)$ affecting the pouring process efficiency was presented. The analysis of the experimental results revealed that the main features of the machine provide the necessary stable shape products and good rheological parameters of these products. The rheological testing indicators allow for a rational approach to the decision to operate a rolling process. At the same time, they improve the quality control methods and have an influence on the design parameters of rollers and modes of chambers. The proposed method not only allows obtaining a predetermined pressure, but also enables defining the optimal shape of the gap, wherein the pressure gradient is constant.
\end{abstract}

Keywords: rolls shape, pouring process, rheological parameters.

\section{INTRODUCTION}

One of the main areas of rational technical support for the forming products process in various industries involves machines with roller operating elements. At present, there is no sufficient variety of designs of roller operating elements, which carry out the technological operation of injection and transportation of a viscous medium. These machines need to be improved and investigated in order to implement new developments in production $[1,2,3]$. Special attention is paid to small-size, efficient machines with controlled processes occurring in the working chamber. While designing such machines, special attention should be paid to the substantiation of the scheme and the appropriate construction solution of the decisions made, since it significantly influences the possibility of such machine manufacturing under the enterprise conditions, the technological capabilities of which are aimed at equipment repair and recovery [3].

At present, the creation of new and refinement of the current technologies cannot be limited to different semi-empirical approaches based on the production experience alone. They must be based on sound fundamental theoretical conclusions. Therefore, the development of the theory of pouring process is carried out by developing the methods of fairly accurate quantitative description of the processes that take place in the medium when it forms in the inter-roll gap and after deformation. A comprehensive study on the behavior of the medium (dough) in the deformation zone during pumping and injection and the establishment of regularities that link the quality parameters with the modes of their deformation, are possible only when using the methods of solid 
medium mechanics $[4,5]$. They allow conducting quantitative assessments of transitions in the environment (dough). In order to analyze these processes, it is necessary to consider the basic rheological properties, conditions on the surface of its contact with the rolls and other factors.

At the same time, a large part of the achievements of the fundamental sciences does not find appropriate application in solving new classes of theoretical and applied problems in the impact of rolls on the environment. These include the prediction of the structure and properties of the environment, defects, stresses, deformation and loading in the treated environment [4].

Thus, due to the multifactorial, complex interdependence, multi-stage production processes, it is not possible without sufficiently rigorous mathematical modeling of the learning processes. The correctness of mathematical models, taking into account the maximum number of influential factors, including the use of previous assumptions in the components of mathematical dependencies, should be based on the results of the studies that are recognized achievements of scientific and technological progress $[5,6]$.

\section{ANALYSIS OF THE AVAILABLE INVESTIGATIONS}

Rolling is a way of processing the dough with plastic deformation, which is the most common method in the baking and confectionery industry when molding products. This method was born in the eighteenth century and over time has reached a high level of perfection. The essence of the process is that the weight of the dough in the working chamber of the machine is compressed passing in the gap between the rotating rolls; thus, it decreases in the cross section and increases in length. The cross-sectional shape is called the profile [7, 9].

The process of passing the dough between the rolls is ensured by the friction forces of the rotating surfaces of the rollers and the dough. Due to the loose surface of the rolls, the dough moves in the gap between them, simultaneously deforming. At the moment of the dough being gripped by each roll, two forces are exposed: normal force and tangent friction force.

In order to develop and improve equipment with roller working bodies and its implementation, a scientifically sound method of calculating the operating parameters flowing in these machines is required. The hydrodynamic theory in the field of processing of food masses has been greatly advanced in the study of the flow of nonNewtonian fluids in the gap of rotating rolls, owing to the scientists O.G. Lunina, Yu. A. Machykhina, S.A. Machihina, V.A. Aretha, V.A. Panfilova and others. They have established that the dough refers to pseudo plastic bodies, the flow of which is described by Ostwald's power law [7, 8, 9].

Accordingly, all scientific work was aimed at forming a viscous environment by rolls. They should influence it in such a way that the losses of raw materials and finished products are minimal and the quality of products is high. This led to the need to ensure full compliance with the processing modes, design forms and parameters of the working roll bodies, as well as the structural and mechanical properties of the viscous medium $[7,10]$.

While working with wheat yeast and pasta dough, the above-mentioned researchers did not fully pay attention to the influence of the gap between the rolls, feed and discharge angle, diameter and geometry of the rolls, changes in torque and energy consumption as well as reliability. The possibility of using this data to form a test piece with the necessary, in terms of practice, shape and size has not been sufficiently studied. It was found that the formation of the dough layer with minimal compaction and preservation of the formholding properties is only ensured by the use of step-by-step rolling of cylindrical rolls [7, 11]. All the data nowadays demand new results, as changes occurred in dough kneading, properties of components and formulations.

It is known that the machines for viscous medium formation should influence it in such a way as to make the loss of raw materials and finished products minimal, and the product quality high. This determines the necessity of ensuring complete correspondence of processing modes, structural shapes and parameters of the operating roller elements, as well as the structural and mechanical properties of viscous medium $[10,11,12]$. This defines the current trends and challenges in designing the roller operating elements in machines of various classes performing work processes: sheeting, injection, mixing, transportation. The largest number of scientific investigations is devoted to the optimization of structural elements profile, the application of composite constructions, the use of modern materials and coatings. 
The specific character of such investigations is associated with an extremely wide range of technological environment properties, the variety of materials used, the distinction of operating conditions. It should be noted that the investigation of the process of a viscous medium rolling by rollers is due to the need for complex use of physical-chemical mechanics of materials, solid-state physics, metallurgy, etc. It should also be pointed out that the traditional methods of viscous medium rolling by rollers (Fig. 1) are based mainly on the combination of deformation load - compression of the working medium in

$$
\left\{\begin{array}{l}
\frac{d \mu}{d t}=f_{\mu}(t, v, T, \rho, P) ; \\
\frac{d v}{d t}=f_{v}(t) ; \\
\frac{d A}{d t}=f_{A}(J, M, t, x, T, \mu, \rho) ; \quad \frac{d J}{d t}=f_{J}(\mu, r, t, v, x, \rho) \\
\frac{d \tau}{d t}=\frac{d P}{d x} ; \frac{d v_{x}}{d x}+\frac{d v_{y}}{d y}=0 ; P=P(x) .
\end{array}\right.
$$

where: $J$ is intensity of rollers mechanical action on medium;

$P(x)$ is pressure; $x$ is coordinate in the direction of shear rate gradient change;

$T$ is temperature;

$M$ is parameter of medium mechanical properties;

$v$ is rollers rotation velocity;

$\mu$ is viscosity;

$A$ is specific sheeting work;

$\tau$ is shear stress. quasi-static and dynamic modes, with the deformation of the entire three-phase medium volume $[2,4,9,11,12]$. Any of the structural schemes with the participation of roller operating elements (Fig. 1) can be mathematically described in the presence of the equations that connect in time by continuous functions the geometric equipment dimensions, properties and the physical-mechanical state of viscous medium, depending on the sheeting, as well as the rollers geometric and climatic characteristics.

The generalized mathematical model of the sheeting process can be presented by the system of differential equations:

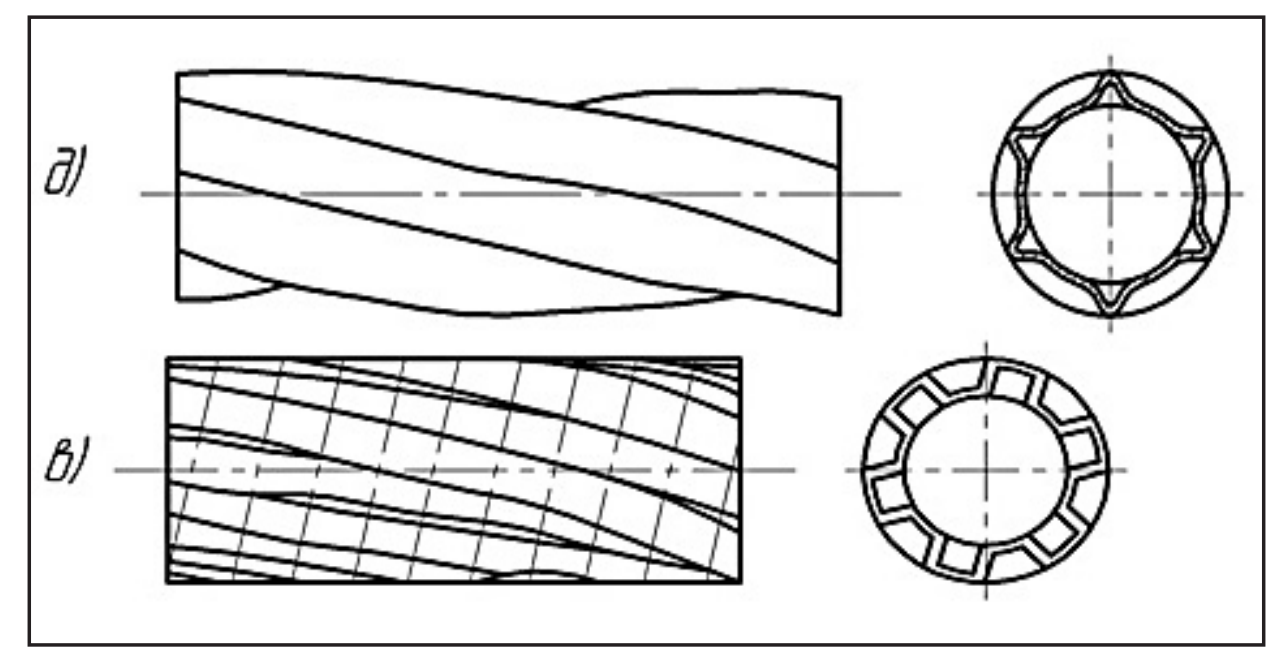

Fig. 1. Scheme of the experimental installation with the roller working bodies: 1 -rollers; 2 - tachometer; 3 -dough; 4 - potentiometer; 5 - thermocouples. 
- the content of raw gluten, $\%-28$;

- resistance gluten compression on the device IDK-1, per. pril. - 54;

- gluten stretch, sm - 14 .

Methods. Any of the structural schemes that were developed and exist with the participation of roller operating elements can be mathematically described by equations connected in time with continuous functions. These include the geometric dimensions of equipment and characteristics of working rollers, as well as the properties and physical-mechanical state of the viscous medium, which depends on the conditions of sheeting.

On the basis of the developed approaches for describing the process of injecting dough in the gap between the rollers, the hydrodynamic theory of calibration of the pseudo plastic fluid was used. This enables to set [19] the quantitative dependencies between the geometric characteristics of the working space (gap) and the properties of materials and processing modes. With the use of this process theory, a number of formulas have been developed to determine the pressure in the gap between the rollers, based on the medium parameters and viscosity.

The characteristics of dough with indestructible structure were investigated by the device RV-8. The experiments were carried out at small shear forces, the magnitude of which was lesser than the boundary shear stress. Every 10-20 seconds the deformation values indicated by the arrow deviation were registered. Knowing the geometric characteristics of the device and measurement data, the shear stress is calculated $\tau^{\prime}$ :

$$
\tau^{\prime}=\tau-\tau_{\kappa}=\frac{\left(m-m_{0}\right) g R_{u \kappa \kappa}}{2 \pi R_{b}^{2} h_{h p}}
$$

where: $m$ - weight of the load that rotates the rotor. It consists of the mass of two weights and masses of cups, $\mathrm{kg}$ (except for the value compensating friction in the bearings); $m_{0}$ is the mass of the load in which the rotor starts to rotate. Accordingly, [10, 13] plastic viscosity was determined:

$$
\mu_{n л}=\left(\tau-\tau^{1}\right) / \gamma
$$

Statement of the problem. Successful solution of the assigned multifaceted tasks to ensure quality operation of the B-4-58 molding machine on the basis of in-depth study of the impact roller working bodies at the stage of formation of bakery and confectionery products. In this regard, the work carried out to determine the influence of various parameters on the structure of the formed "Podolskiy" dough bagel. It should be noted that to ensure more accurate operation of the moulding machine, a partial absence of gas inclusions in the dough is of great importance. Under the action of the rolls, the dough is subjected to repeated staving and rolling. This action leads to the dough compaction, due to the allocation of gas in it and has quite a significant value to stabilize pressure at its discharge and compliance the optimal modes of operation of the rolls. Therefore, with the passage of the technological process, it is necessary to apply the correct method of determining the required design parameters for designing a roller working bodies.

In the research, the authors assumed that in the dough between the rolls is in active connection of the individual particles. An important rheolo-mechanical parameter characterizing the process of the discharge under the conditions of uniform compression, is the pressure generation in the dough. Pressure is a constant for a given machine and has its limits. This is because the compression of the dough rolls leads to a decrease in pore volume and the elastic deformation of the particles. Therefore, in our experiments, the pressure was not taken into account and the indicators that influence more on hisotory property that affects the structure of the bagel were taken note of. Table 1 presents the indicators of the overall technological quality indicators of the test workpiece. They provide an opportunity to judge the compliance process for a "Podolskiy" bagel.

Let us consider the feeding unit of the molding machine (Fig. 2) consisting of dough loading hopper 1, discharge chamber 2 made of two ribbed rollers 3 installed in parallel with the possibility of dough passing between them. Any of the existing and developed structural schemes with the participation of roller operating elements can be mathematically described by equations that are connected in time by continuous functions. We will take into account the geometric dimensions and characteristics of rollers, as well as the properties and some physical-mechanical characteristics of viscous medium. The new rollers surface shape proposed by the authors differs by cylindrical-screw grooves with constant along the length of screw lines step $[13,14]$.

The determination of the medium movement peculiarities in the gap between the rollers is quite 


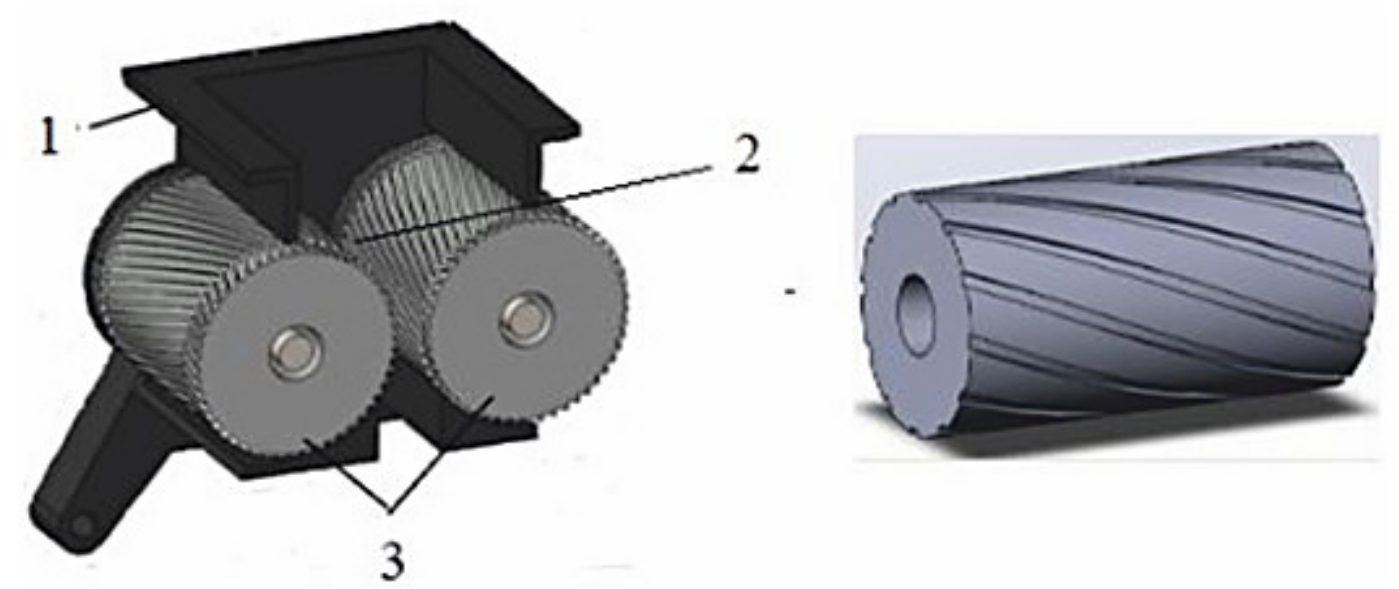

Fig. 2. Cross section of dough pump assembly: 1.working chamber 2.screw rolls

complicated and at the same time, an essentially important problem $[1,15]$. This is due to the need for various types of calculations for forming working rollers determining their effect on the medium. In order to solve such class of problems, it is reasonable to involve the logic algebra and the set theory $[1,2,16]$. At the same time, it is necessary to take into account the medium properties. This enables to consider the arrangement as the ordered set of operating links, and their various combinations, as logical relations of the set elements $[2,16,17]$. In general case, these tasks are more extreme. While choosing the best option for determining the influence of roller structural parameters, the prioritization method, i.e. the options qualitative assessment, is chosen. The solution of the set tasks is possible on the basis of careful investigation of the essence of the process occurring during dough sheeting by rollers.

The irregularity of the velocity of the movement of the medium (bagel yeast wheat dough) is due to the nature of the flow. Therefore, a significant factor in increasing the effectiveness of the intensive rolls on the environment is the uniform pumping of its elementary volumes coming from the working chamber of the machine. On the basis of the assumption that the specific flow of the medium is the same for all elementary volumes of mass that are in the gap between the rolls, the viscosity remains constant and it was not taken into account in the calculations.

The objective of the paper was to determine the influence of the rollers geometry on the injection quality under the condition of horizontal dough sheeting.

\section{RESULTS AND DISCUSSION}

The analysis of papers $[7,13,17,18,20]$ proved that the medium velocity unevenness (bread ring yeast wheat dough) is due to the flow nature. Therefore, a significant factor in increasing the effectiveness of the rollers intensive action on the medium is the uniformity of sheeting its elementary volumes running out from the machine working chamber. Taking into account the assumptions that the specific medium flow is the same for all the elementary mass volumes located in the gap between the rollers, the viscosity remains constant and it was not considered in the calculations.

Schematically, the process of dough sheeting is shown in Figure 3. The rollers of similar diameters rotate towards each other under continuous medium motion (wheat dough).

The inertial properties of viscous medium are omitted. The type of the function describing the consumption rate is as follows:

$$
J(\operatorname{Par}, A, B)=e^{A \cdot(B-P a r)^{2}}
$$

Assuming that $J(P a r)=G$, the unknown coefficients in equation (1) $A$ and $B$ are determined for each case with the smallest squares method. Three cases are considered.

\section{THEORETICAL CALCULATION RESULTS FOR THE CASE OF THE GAP EFFECT}

Let us consider the change in the parameters of the belonging function $J_{\mu}(h)$ and the theoretical consumption rate $G(h)$ of viscous medium on the rollers from the gap $h$ between them along the 


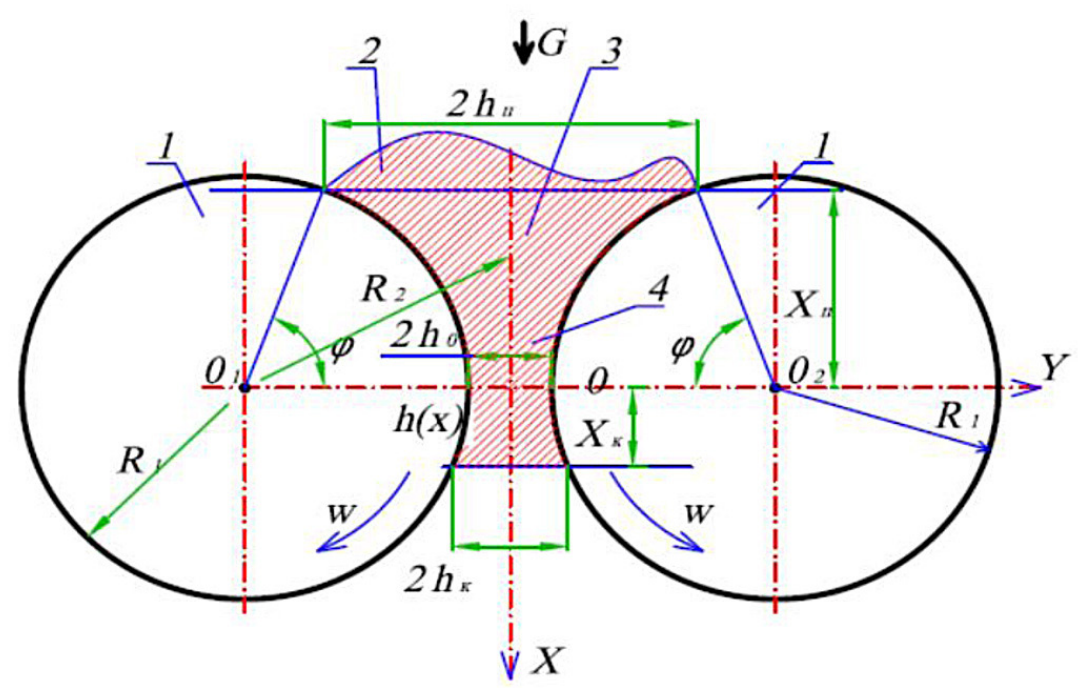

Fig. 3. Profile scheme of dough sheeting between the rollers: 1 - rotating rollers with radius $R$, half the distance between them is $h_{0} ; 2$ - dough flow of half thickness $h$ at the working chamber entrance; 3 - sheeted dough after rollers passing

length. We form the consumption distribution $J_{\mu}$ on the gap $h$, which varies from $1 \ldots 5.5 \mathrm{~mm}$ and the probability of the given factor availability. The highest probability 0.92 is investigated for $h=3.6 \mathrm{~mm}$. While moving from the centre, it decreases to 0.1 .

$$
J_{\mu}=\left(\begin{array}{l}
1 \ldots \ldots .2 .5 \ldots \ldots 3 \ldots \ldots 3.6 \ldots .4 .5 \ldots \ldots 5 \ldots .5 .5 \\
0.1 \ldots 0.26 \ldots 0.48 \ldots 0.92 \ldots 0.4 \ldots 0.22 \ldots 0.1
\end{array}\right)
$$

For the practical use of expression (1), you need to set the values $A$ and $B$. Let us apply the smallest errors operator to determine $A$ та $B$. Let us also apply the smallest error operator of MathCAD program. We use the Given - Minerr block.

$$
\mathrm{n}=\operatorname{cols}\left(\mu_{\mathrm{h}}\right) \mathrm{n}=7 \mathrm{~B}=3.60 \mathrm{~A}=-0.01
$$

Given

$$
\begin{gathered}
\sum_{i=0}^{n-1}\left(\mu_{h_{1, i}}-\mu\left(\mu_{h_{0, i}} A, B\right)\right)^{2}=0 \\
\left(\frac{A_{h}}{B_{h}}\right)=\operatorname{Minerr}(A, B) \\
\frac{A_{h}}{B_{h}}=\left(\frac{-0,954}{3.718}\right) \\
M:=\max \left[\left|\left(\mu_{m}{ }^{T}\right)^{\langle 1\rangle}\right|\right] m:=\min \left[\left(\mu_{h}{ }^{T}\right)^{\langle 1\rangle}\right] \\
M=0.92 m=0.1 \\
\boldsymbol{A}_{\boldsymbol{h}}=-\mathbf{0 . 9 5 4 ,} \boldsymbol{B}_{\boldsymbol{h}}=\mathbf{3 . 7 1 8}, \boldsymbol{i}=\mathbf{0} \ldots \boldsymbol{n}-\mathbf{1 , h = 1 , 1 . 1 . . 6} \\
\mu_{h}(h):=\mu\left(h, A_{h}, B_{h}\right)
\end{gathered}
$$

$$
\boldsymbol{h}_{\boldsymbol{0}}=1 \boldsymbol{h}_{\boldsymbol{m}}=6 \boldsymbol{c}=2.9
$$

According to the calculation results, the dependence of the consumption rate of viscous mass according to (1) is as follows:

$$
G(h)=e^{-0.95 \cdot(3.72-h)^{2}}
$$

Let us construct the graphs of functions $(\mu h)$, $[G(h)]$ according to the data (2) Figure 4.

In Figure 3 the results of theoretical calculations are represented by the curve, while the experimental data - by points. The analysis of the curve nature shows that the gap size affects the consumption and, accordingly, the process quality. The rational value of the gap between the rollers, which ensures high-quality dough rolling sheeting, is within $3-4.2 \mathrm{~mm}$. It should be noted that the comparisons of experimental and research data are well correlated.

Some characteristics of dough and blanks for different gap values and rollers diameter are represented in Table 1. These data prove the rational values of the gap between the rollers obtained on the basis of the theoretical calculation.

At the next stage, the belonging function of consumption rate $G G(h)$ along the rollers plane as the normalized quantity was investigated. Interval of argument change is $h: h_{0}=1 \ldots h_{m}=7$ :

\section{$G G(h) d h$}

$$
\begin{aligned}
S=\int_{h_{0}}^{h_{m}} & \\
& S=9.664
\end{aligned}
$$




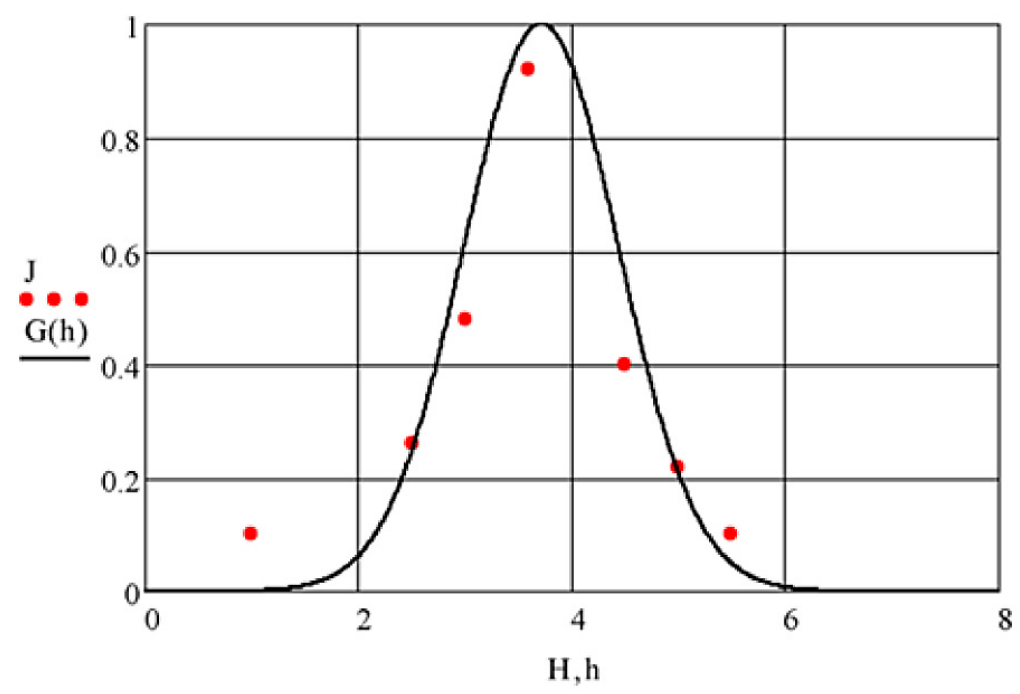

Fig. 4. Function of medium consumption belonging along the rollers plane

The dependence of the belonging function $G G(h)$ is shown in Figure 4. function.

$$
G G(h)=\mid \begin{array}{lr}
C(1-G(h)) \text { if } 0 \leq h \leq 7 \\
0 & \text { otherwise }
\end{array}
$$

The obtained results prove the correctness of the initial conditions assumed for the determination of the dependence of the gap between the rollers along the length on the working medium consumption.

\section{THEORETICAL CALCULATION RESULTS FOR THE CASE OF ROLLER RADIUS PARAMETERS ACTION}

The investigation of the belonging function parameters for the consumption rate of the viscous medium between the rolls relatively to its radius was carried out. The position of point $\mathrm{O}$ (Fig. 3) was fixed in mm. The function GG(r) in the rollers plane was searched as the normalized quantity. The calculation principle was the same as in paragraph 1.

$$
F_{\mu}=\left(\frac{155}{0.14} \frac{158}{0.36} \frac{161}{0.68} \frac{167}{0.66} \frac{170}{0.32} \frac{175}{0.11}\right)
$$

$$
\mathrm{n}:=\operatorname{cols}(\mathrm{F} \mu) \quad \mathrm{n}:=7 \quad \mathrm{~B}:=164 \mathrm{~A}:=-0.1
$$

Given $\sum_{i=0}^{n-1}\left(F_{\mu 1, i}-G\left(F_{\mu 0, i}, A, B\right)\right)^{2}=0$

$$
\begin{aligned}
& \left(\frac{A_{r}}{B_{r}}\right)=\operatorname{Minerr}(A, B) \\
& \frac{A_{r}}{B_{r}}=\left(\frac{-0,032}{163.862}\right)
\end{aligned}
$$

We obtain the formula of the medium consumption dependence on the parameter $r$

$$
G G(r)=0.99 \cdot e^{-0.032 \cdot(163.862-r)}
$$

(listing 2)

$$
n=\operatorname{cols}\left(\mu_{m}\right) \quad n=7, B:=164, A:=-0.1
$$

Given

Table 1. Experimental data of dough properties after the rollers and molding device action

\begin{tabular}{|c|c|c|c|c|c|c|c|c|}
\hline No. & $\begin{array}{c}n, \\
\text { rev./min. }\end{array}$ & $\begin{array}{c}m_{1}, \\
\mathrm{~kg} .\end{array}$ & $\begin{array}{c}\text { G, specific } \\
\text { gas formation }\end{array}$ & $K$ & $\begin{array}{c}h, \text { gap between } \\
\text { rollers, } \mathrm{mm} .\end{array}$ & $W$ humidity & $\begin{array}{c}\rho \\
\mathrm{kg} / \mathrm{m}^{2}\end{array}$ & Temperature \\
\hline 1. & 1.2 & 0.0078 & 10 & 1.57 & 1 & 31.6 & 1.12 & 27 \\
\hline 2. & 1.2 & 0.0077 & 10 & 1.57 & 2 & 31.6 & 1.12 & 27 \\
\hline 3. & 1.2 & 0.0079 & 10.2 & 1.57 & 2.5 & 31.7 & 1.12 & 27.3 \\
\hline 4. & 1.2 & 0.0087 & 10.2 & 1.57 & 3 & 31.7 & 1.123 & 27.2 \\
\hline 5. & 1.2 & 0.0097 & 10.3 & 1.57 & 3.5 & 31.7 & 1.123 & 27.3 \\
\hline 6. & 1.2 & 0.0099 & 10.2 & 1.57 & 4.5 & 31.6 & 1.124 & 27.4 \\
\hline 7. & 1.2 & 0.0098 & 10.5 & 1.57 & 5.5 & 31.7 & 1.12 & 27.4 \\
\hline
\end{tabular}




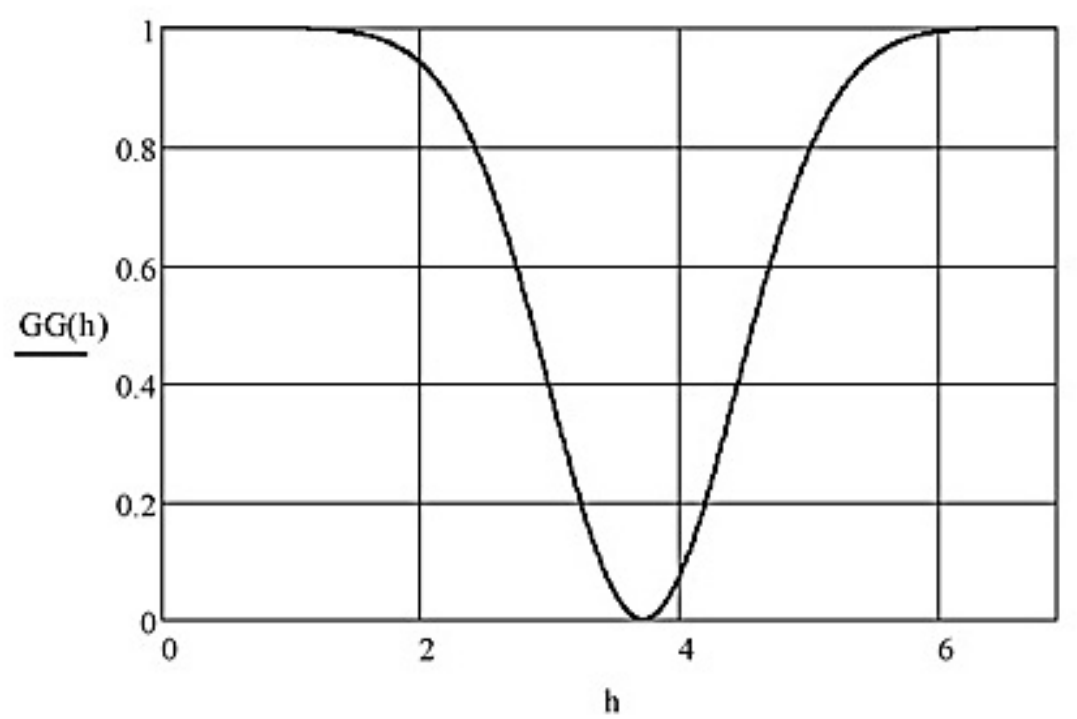

Fig. 5. Function $\mathrm{GG}(\mathrm{h})$ viscous medium consumption on the gap size

$$
\sum_{i=0}^{n-1}\left(\mu_{m_{1, i}}-\mu\left(\mu_{m_{0, i}}, A, B\right)\right)^{2}
$$

$$
\begin{gathered}
\left(\frac{A_{r}}{B_{r}}\right)=\operatorname{Minerr}(A, B) \quad \sum_{i=0}^{n-1}\left(\mu_{m_{1, i}}-\mu\left(\mu_{m_{0, i}} A, B\right)\right)^{2} \\
A_{r}=-0.032 B_{r}=163.862 m m:=\min \left[\left(\mu_{m}{ }^{T}\right)^{\langle 1\rangle}\right] \mathrm{m}=0.11 \\
\mu_{m}(r):=\mu\left(r, A_{r}, B_{r}\right) n:=\operatorname{col}\left(\mu_{m}\right) \\
I=0 \ldots n-1 ; r=155,155.1 \ldots 175
\end{gathered}
$$

Let us carry out the normalization test according to the standard and select $C C$ in the belonging function formula choosing the interval for argument change the $r: r_{0}=155$ to $r_{m}=175$.

$$
\begin{gathered}
r_{0}=155 r m=175 S=9.689 C C=0.99 \mu_{m} r=\mathrm{CC} \mu_{\mathrm{m}}(r) \\
S=\int_{r_{0}}^{r m} \mu_{m}(r) d r
\end{gathered}
$$

The consumption belonging to function $G G(r)$ in the rollers plane is found as the normalized quantity. Let us carry out the normalization test according to the standard $G=9.7$ and select $C C$ in the belonging function formula, choosing the interval for argument change $r: r_{0}=155$ to $r_{m}=175$. We obtain the formula of the medium consumption dependence on parameter $r$ :

$$
G G(r)=0.99 \cdot e^{-0.032 \cdot(163.862-r)}
$$

On the basis of carried out calculations, let us construct the function graphs $F\left(\mu_{h}\right),[G(h)]$ according to the data (5) in Fig. 5. In Figure 5, the theoretical values are represented by the curve, and experimental data by the points. According to the obtained results rational values of the roller diameters from 162 to $168 \mathrm{~mm}$.

\section{COMPLEX EFFECT OF GAP $H$ AND RADIUS-VECTOR $R$}

The joint effect of two factors was carried out through the logical product of the belongings by minimizing two determined functions along twodimensional area of two factors $r$ and $h$.

$$
\mathrm{G}(\mathrm{h}, \mathrm{r})=\min (\mathrm{GG}(\mathrm{h}), \mathrm{GG}(\mathrm{r})),
$$

In order to ensure the calculations, the problem of coarse grain argument was solved by choosing number $N$ - the number of points of the change range division of the range $r$ and $h$.

$N=20 i=0 \ldots N j=0 \ldots N r_{1}=155 \quad r_{2}=175 h_{1}=\mathrm{O} \quad h_{2}=7$

$$
M_{i, j}=\mu_{r} h\left(r_{i}, h_{j}\right)
$$

For practical reasons we choose submatrix $M$ of the entire $\operatorname{array}\left(r_{i}, h_{j}, M_{i j}\right)$

$$
\begin{gathered}
\mathrm{r}_{\mathrm{i}}=\mathrm{r}_{1}+\left(\mathrm{r}_{2}-\mathrm{r}_{1}\right) \mathrm{i} / \mathrm{N} \\
h_{j}=h_{1}+\left(h_{2}-h_{l}\right) j / N
\end{gathered}
$$

The functional dependences of the medium consumption, depending on $r$, and $h$ are shown in Figure 7.

$$
M_{m}=\min (M)=0.019
$$

$$
M=\text { submatrix }(M, 0,20,5,15)
$$

The lower and upper faces correspond to the surface and inter-surface medium motion 


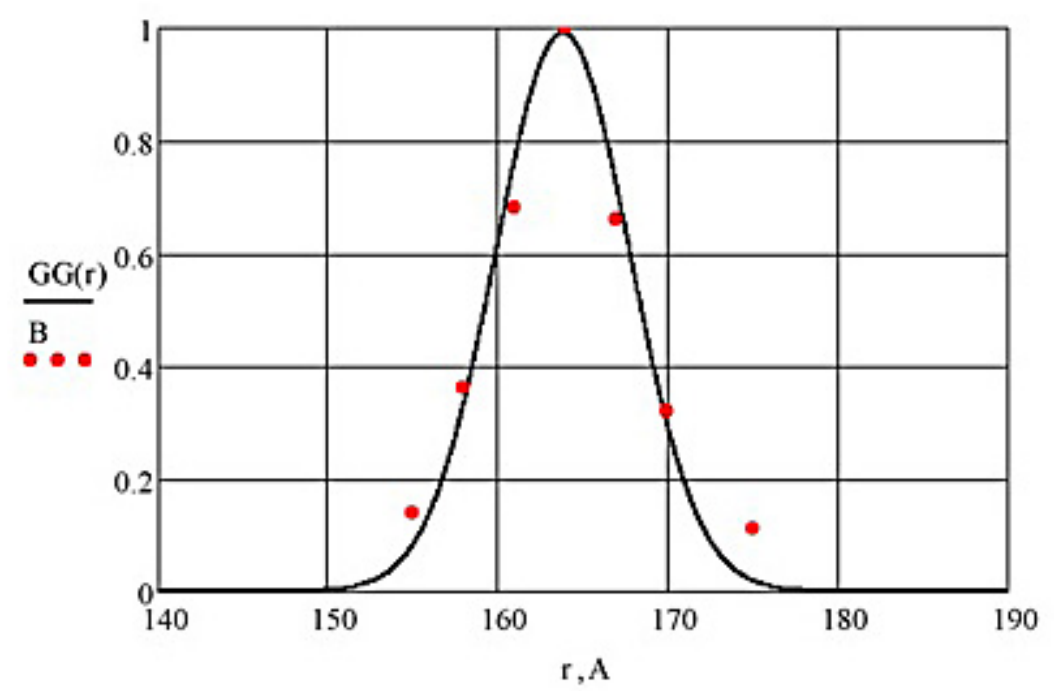

Fig. 6. Medium consumption dependence on radius-vector medium point relatively to roller
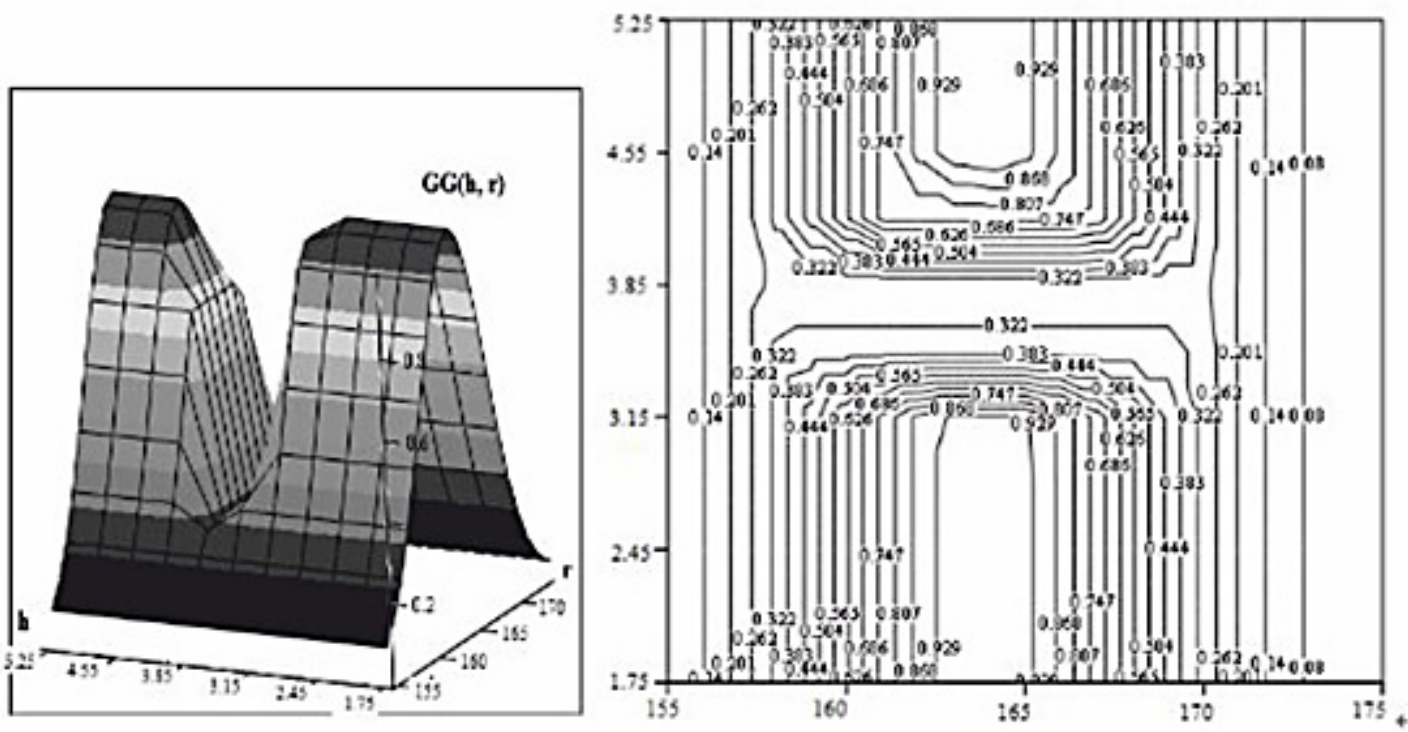

Fig. 7. Graphs of fizzy medium consumption function: a- three-dimensional; b- order line

between the rollers, respectively [3, 8].The analysis of the obtained results shows that the largest medium movement occurs near the rollers surfaces, which depends on $\mathrm{h}$ and changes in the direction of the central inter-roller axis reduction. This special medium movement can be divided into two stages. The first one is the adhesive medium contact with the roller surface $[20,21]$. The speed changes along the roller surface. At this stage of the movement, the determining factor is the rollers diameter of. The second stage of the movement is the inter roller variable medium motion.

\section{CONCLUSIONS}

On the basis of the proposed method for the investigation of the influence of the roller radius and the gap between them on the dough consumption, the functional dependences for calculating the rational roller diameter and the gap between them were substantiated. Thus, to ensure rational dough consumption, the rollers diameter range is $155-165 \mathrm{~mm}$, the gap $-1.5-3.4 \mathrm{~mm}$. It is determined that the mass is moving more intensely near the rollers surface due to the adhesion influence. The proposed method of investigation the nature 
of the medium motion can be used while designing the roller injectors (dough dividing machines, molding machines), while doing, mixing, and etc.

Determination of $r$ and $h$ parameters enables to reduce energy consumption, increase efficiency and minimize the material loss. The analysis of the experimental results proved that the machine design features provide the necessary stable products form and sufficient rheological products parameters. This improved the quality control methods and control design parameters of rolls and modes in the camera. The proposed method of calculation enables to determine the rational gap shape, to provide constant pressure gradient.

\section{REFERENCES}

1. Mitsoulis E., Hatzikiriakos S.G. 2009. Rolling of bread dough: Experiments and simulations. Food and bioproducts processing.

2. Bloksma A.H. 1990. Dough structure, dough rheology, and baking quality. Cereal Foods World, 35(2), 237-244.

3. Holzapfel G.A. (2000). Nonlinear Solid Mechanics: A Continuum Approach for Engineering, New York.

4. Malkin A.Y., Isayev A.I. 2006. Rheology: conception, methods, application. ChemTec Publishing, Toronto, $474 \mathrm{p}$.

5. Mitchell T. A. 1984. Dough mixer controls for the mechanical dough development process, Proceedings of the International Symposium on Advances in Baking Science and Technology, Kansas State University, Manhattan, KS.

6. Bloksma A. Niemann W. 1975. The effects of temperature on some rheological properties of wheat flour doughs. Journal of Texture Studies, 6(3), 343-361. https://doi.org/10.1111/j.1745-4603.1975.tb01130.x

7. Machikhin Y.A. 1992. Formation of food masses.

8. Chakrabarti S. Bergström J.S., Lindskogand E., Sridhar T. 2010. Computational modeling of dough sheeting and physical interpretation of the non-linear rheological behavior of wheat flour dough, pp. 278-288, doi:10.1016/j.jfoodeng.2010.04.010

9. Chu S.G. 1989. Handbook of pressure sensitive adhesive technology, Chapter 8. New York: Van Nostrand Reinhold.
10. Paul Singh R., Kenneth J., Valentas E., Handbook of food engineering practice. E. Rotstein (Ed), Includes bibliographical references and index. (alk. paper).

11. Khamis M. 2014. Characterization and evaluation of heat treated wheat flours: dissertation theses. Manhattan, Kansas: Kansas State University, p. 160. https://core.ac.uk/download/pdf/33353737.pdf.

12. Declarative Patent for utility model Ukraine. Dough feeding unit of the molding machine. Patent owner: Stadnyk I. A 21C 3/10 (2006.01) Ukraine. Patent no.111058., 25.10.16.

13. Declarative Patent for utility mo Ukraine. Dough feeding unit of the molding machine. Patent owner: A.Derkach, I., Stadnyk, O. Stadnyk.A 21C 3/10 (2006.01) Ukraine. Patent no.111061. 25.10.2016.

14. Muratova E., Smolihina P. 2015. Reologiya konditerskih mass: monografiya: FGBOU VPO«TGTU», vol. 6, p. 188 http://oreluniver.ru/e/chair/thkimp/ news/Sbornik_2015.pdf

15. Nikolaev B. 1976. Measuring of structural and mechanical properties of flour dough. Food Industry, 83-127.

16. Obolkina V., Skrypko A., Kyianytsia S. 2016. The scientific approach to the creation of technologies pastry recreational purposes using flour with malt oats. Food industry, vol. 19, p. 73-78. Available at: http://nbuv.gov.ua/UJRN/Khp_2016_

17. Pareyt B. 2013. Impact of mixing time and sodium stearoyllactylate on gluten polymerization during baking of wheat flour dough. Food Chemistry, 141, 4179-4185.

18. Paul Singh R., Kenneth J., Valentas E. Handbook of food engineering practice. E. Rotstein (Ed.), Includes bibliographical references and index. (alk. paper).

19. Stadnyk D.I., Katarzyna S. 2016. Determinanion of power output and impact on the rheological parameters of raw materials during rolling. Agricultural Engineering, 20, 5-12.

20. Stadnyk I., Novak L, Matenchuk L. 2018, Global rheological approach to the quality of medium injected by the rollers. Potravinarstvo Slovak Journal for Food Sciences, 11, 234-245.

21. http://www.potravinarstvo.com/journal1/index. $\mathrm{php} /$ potravinarstvo/article/viewFile/867/pdf

22. Stadnyk I.Y., Piddubny V., Karpyk H., Yeremeeva O. 2018. Features of heat transfer in the environment when it is sprayed with rotary rollers. Potravinarstvo Slovak Journal for Food Sciences, 12(11), 824-835. https://doi.org/10.5219/977. 